\title{
SYSTEMS OF EXTREMALS IN THE CALCULUS OF VARIATIONS.
}

BY PROFESSOR EDWARD KASNER.

(Read before the American Mathematical Society, December 29, 1906.)

THE extremals connected with any problem in the calculus of variations are the curves or surfaces or higher manifolds which satisfy the first of the conditions necessary for a maximum or minimum value. In the case of the integral of simplest type

$$
\int F\left(x, y, y^{\prime}\right) d x
$$

this condition is given by the Euler-Lagrange equation

$$
\frac{d}{d x} F_{y^{\prime}}-F_{y}=0,
$$

where the subscripts indicate partial differentiation. The expanded equation is

$$
F_{y^{\prime} y^{\prime}} y^{\prime \prime}+F_{y^{\prime} y} y^{\prime}+F_{y^{\prime} x}-F_{y}=0 .
$$

The extremals here form a doubly infinite system of plane curves. Darboux's well-known investigation of the inverse problem shows that the system is entirely general *: Any doubly infinite system of curves may be regarded as the system of extremals belonging to some integral of type (1).

In this respect the simplest type of problem is unique. In all the more complicated integrals, involving either derivatives of higher order, or more than one dependent or independent variable, the Euler-Lagrange equations are of special form, and hence the system of extremals (curves or other manifolds) must possess peculiar geometric properties. Our object is to give an example, apparently the simplest, of such a property.

Consider the plane problem of second order connected with the integral

$$
\int F\left(x, y, y^{\prime}, y^{\prime \prime}\right) d x .
$$

\footnotetext{
* Darboux, Théorie des surfaces, vol. 3, no. 604.
} 
The extremals form a quadruply infinite system of curves with the equation

$$
\frac{d^{2}}{d x^{2}} F_{y^{\prime \prime}}-\frac{d}{d x} F_{y^{\prime}}+F_{y}=0 .
$$

This is found to be of the form

$$
y^{\mathrm{IV}}=A y^{\prime \prime \prime 2}+B y^{\prime \prime \prime}+C,
$$

where $A, B, C$ involve only $x, y, y^{\prime}, y^{\prime \prime}$. It will not be necessary, for our purpose, to have the values of these coefficients in terms of the partial derivatives (of first, second, and third order) of $F$.

An extremal is determined when the initial values of $x, y$, $y^{\prime}, y^{\prime \prime}, y^{\prime \prime \prime}$ are assigned. If only $x, y, y^{\prime}, y^{\prime \prime}$ are given, that is, a curvature element, there will be $\infty^{1}$ corresponding curves. For each of these at the common point construct the osculating conic. The locus of the centers of the conics so obtained may be termed the central locus corresponding to the given curvature element. What is the character of the central locus?

The general formulas for the center of the conic osculating a given curve at a given point may be obtained most readily by means of a theorem of Cayley : the center is determined by the intersection of consecutive axes of deviation.* Using coordinates $X, Y$ referring to the given point as origin, we find the equation of the axis of deviation at the given point to be

where

$$
Y / X=m,
$$

$$
m=\frac{y^{\prime} y^{\prime \prime \prime}-3 y^{\prime 2}}{y^{\prime \prime \prime}} .
$$

At the consecutive point the equation becomes

$$
\frac{Y-d y}{X-d x}=m+m^{\prime} d x,
$$

where

$$
m^{\prime}=\frac{y^{\prime \prime}\left(3 y^{\prime \prime} y^{\mathrm{IV}}-5 y^{\prime \prime \prime 2}\right)}{y^{\prime \prime \prime 2}} .
$$

* Salmon-Fiedler, Höhere Kurven, p. 468. The point in question is sometimes termed the center of deviation. 
The intersection of (7) and (8) is the required center

$$
X=\frac{3 y^{\prime \prime} y^{\prime \prime \prime}}{5 y^{\prime \prime \prime 2}-3 y^{\prime \prime} y^{\mathrm{IV}}}, \quad Y=\frac{3 y^{\prime \prime}\left(y^{\prime} y^{\prime \prime \prime}-3 y^{\prime \prime 2}\right)}{5 y^{\prime \prime 2}-3 y^{\prime \prime} y^{\mathrm{IV}}} .
$$

To apply these general formulas to the question proposed, we first solve them with respect to $y^{\prime \prime \prime}, y^{\mathrm{IV}}$, obtaining

$$
y^{\prime \prime \prime}=\frac{-3 y^{\prime \prime 2} X}{Y-y^{\prime} X}, \quad y^{\mathrm{IV}}=\frac{3 y^{\prime \prime 2}\left(5 y^{\prime \prime 2} X+Y-y^{\prime} X\right)}{\left(Y-y^{\prime} X\right)^{2}} .
$$

Substituting these values in (6), we find the relation

$$
\begin{aligned}
& 3 y^{\prime \prime 2}\left(Y-y^{\prime} X\right) \\
& \quad=3 y^{\prime \prime 3}\left(3 y^{\prime \prime} A-5\right) X^{2}-3 y^{\prime \prime 2} B X\left(Y-y^{\prime} X\right)+C\left(Y-y^{\prime} X\right)^{2} .
\end{aligned}
$$

Here $A, B, C$ have fixed values since the initial element $x, y, y^{\prime}, y^{\prime \prime}$ is supposed to be given. The central locus, defined by (11), is thus a conic. Furthermore it passes through the given point, the origin of the $X, Y$ system ; and its tangent at that point, namely,

$$
Y-y^{\prime} X=0,
$$

has the direction of the given element. We state the result as follows:

The extremals connected with any plane problem of the second order, that is, with any integral of type (4), form a quadruply infinite system of curves such that $\infty^{1}$ are determined by a given element of curvature. The locus of the centers of the conics which osculate these $\infty^{1}$ curves at their common point is a conic tangent to the given element.

From the discussion it is seen that the result is not completely characteristic since it holds for all systems whose differential equation is of form (6), and such a system need not be a system of extremals. In the latter case the functions $A, B, C$ are connected by rather complicated relations derived by expressing the fact that certain partial differential equations are consistent.

That the property stated is, however, characteristic of the form (6) may be shown as follows. By assumption, the central locus for any element of curvature is to be a conic touching the 
element. Its equation is then

$$
Y-y^{\prime} X=a X^{2}+b X Y+c Y^{2} .
$$

Substituting the general values given in (9), and reducing, we find that $y^{\mathrm{IV}}$ is quadratic in $y^{\prime \prime \prime}$. The equation is therefore of the form (6).

If it is required merely that the central locus shall be a conic, without any restriction as to position, then a more general class of differential equations of the fourth order is obtained, involving five instead of three arbitrary functions of $x, y, y^{\prime}, y^{\prime \prime}$. In particular, if the conic is to pass through the given point, the resulting equation is of the form

$$
y^{\mathrm{IV}}=\alpha y^{\prime \prime \prime 3}+\beta y^{\prime \prime \prime 2}+\gamma y^{\prime \prime \prime}+\delta .
$$

An interesting special case of systems of type (6) is obtained by imposing the condition that for every curvature element the conic (11) shall degenerate. This is so only when $A$ has the special form

$$
A=\frac{5}{3 y^{\prime \prime}} \text {. }
$$

The conic then breaks up into the trivial part (12) and the proper part

$$
3 y^{\prime \prime 2}(B X+1)-C\left(Y-y^{\prime} X\right)=0 .
$$

In the case of a system of extremals the value of $A$ in terms of the integrand function $F$ is

$$
A=-\frac{F_{y^{\prime \prime} y^{\prime \prime} y^{\prime \prime}}}{F_{y^{\prime \prime} y^{\prime \prime}}}
$$

By comparing (15) and (17) and integrating, we find

$$
F=f_{1}\left(x, y, y^{\prime}\right) y^{\prime \frac{1}{3}}+f_{2}\left(x, y, y^{\prime}\right) y^{\prime \prime}+f_{3}\left(x, y, y^{\prime}\right),
$$

where the $f$ 's are arbitrary functions.

Hence the only case in which the system of extremals connected with an integral (4) is such that the central loci are straight lines is that in which the integrand function is of the form (18).

Columbia University. 